\title{
Thickness dependence of higher-order Laue zone line positions at strongly dynamic zone axes
}

\author{
John Mansfield ${ }^{a}$, David Bird ${ }^{b}$ and Martin Saunders ${ }^{b}$ \\ ${ }^{a}$ North Campus Electron Microbeam Analysis Laboratory, University of Michigan, Ann Arbor, MI 48109-2143, USA \\ ${ }^{b}$ School of Physics, University of Bath, Claverton Down, Bath, UK
}

Received 20 August 1992

\begin{abstract}
Simulation of bright-field convergent-beam electron diffraction (CBED) discs has revealed that, at zone axes where there are two strongly excited branches of the zero-layer dispersion surface that may interact with the HOLZ, the deficiency HOLZ line positions should be sensitive to changes in thickness. Examination of experimental patterns at carefully chosen accelerating voltages have corroborated the findings of the calculations. The effects are subtle; however, they are large enough to introduce significant errors into lattice parameter or strain measurements made by monitoring HOLZ positions. The magnitude of the effect is considerably reduced at zone axes where the dynamic diffraction is weaker (e.g. $\langle 114\rangle$ ), so much so that, with care, these axes are suitable for use in strain or lattice parameter determination.
\end{abstract}

\section{Introduction}

The use of the positions of higher-order Laue zone (HOLZ) lines, visible in the bright-field discs of convergent-beam electron diffraction (CBED) patterns, to measure the local lattice parameters of phases in the transmission electron microscope was first discussed by Jones et al. [1]. They were careful to note, however, that while HOLZ lines are comparatively insensitive to specimen thickness, due to the long extinction distances associated with HOLZ diffraction, there were a number of factors that could affect the visibility, width and apparent position of the lines. They showed that specimen thickness played an important role in the width of the HOLZ line detail. Optimum line widths are obtained when one balances the narrowing of the lines with increasing thickness with their visibility as a function of increasing thermal diffuse scattering. Jones et al. also noted that line shifts and broadening occurred when the zero-layer fringes of certain simple string zone axes interacted with bright-field HOLZ lines. They did, however, in spite of these caveats, test the feasibility of measuring the lattice parameter of phases by measurement of the HOLZ line positions. They used the HOLZ detail in the bright-field disc of the silicon $\langle 111\rangle$ zone axis to test how well absolute determination of the lattice parameter of a sample compared with X-ray methods. They noted that the techniques showed promise; however, they were not totally satisfied. The simulation of the HOLZ line positions via a kinematical approximation yielded poor agreement with X-ray results and there was evidence that their measurements were being affected by dynamical thickness dependence of the HOLZ line positions. Later, Steeds noted that it was more reasonable to measure the changes in lattice parameter with respect to a known reference with the same crystal structure [2].

Since that time there have been a large number of studies that have exploited the technique and it has become one of the "standard" applications of CBED in the analytical electron microscope (AEM); unfortunately the warnings made by Jones et al. [1] are not always heeded [3-6]. The technique requires the use of a "standard" 
of "known" lattice parameter. In order to limit the dynamic diffraction effects, the standard and the unknown must have similar strength projected potentials along the zone axis under study, i.e. using gold as a standard to measure changes in nickel would not produce accurate results [2]. Typically, patterns are recorded from the standard, and the HOLZ line detail in the bright-field disc is simulated in a computer using the kinematic approximation. The microscope accelerating voltage is used as a "free" parameter to obtain a best fit of the pattern to the "known" lattice parameters. Patterns from the unknown phase are recorded and the HOLZ line positions are simulated kinematically, the lattice parameters being varied to obtain the best fit to the experimental patterns. Lin et al. [7] discussed the errors that arise from using simple kinematic theory for lattice parameter measurements, and proposed a correction that accounts for the movement of the HOLZ line away from its kinematic position which is caused by the shift of the dynamical dispersion surface away from its kinematic equivalent. Generally users of this technique do not consider the thickness of the sample to be a parameter in determining the HOLZ line position. This paper describes the results of both computer simulations and cold-stage experiments in the AEM which show that the apparent positions of HOLZ lines at certain zone axes are strongly dependent on thickness due to dynamical zero-layer diffraction effects.

\section{Calculations and experimental techniques}

The observations reported in this paper were the result of some preliminary studies to extract further information from CBED patterns other than symmetries and lattice parameters. Brightfield CBED patterns were calculated by a computer program that calculates the diffracted beam intensities by matrix diagonalization of the many-beam equations. The program is written in FORTRAN 77 and has evolved from code originally developed by Baker [8]. The program has been simply modified to run on a number of processing units $N$ and each unit calculates
$1 / N$ th of the pattern. For this study we have employed a parallel computing cluster maintained by the University of Michigan ComputerAided Engineering Network. This cluster currently consists of 8 IBM RS/6000 Model $320 \mathrm{H}$ workstations connected by a localized ethernet network. The parallel processing is performed under the Parallel Virtual Machine system, a parallel processing environment developed by Oak Ridge National Laboratory [9]. The parallel operation of the program allows the simulation of well converged patterns (which include 37 zerolayer reflections and 33 reflections from the FOLZ), with a high angular resolution (up to $601 \times 601$ pixels), that are also corrected for absorption [10]. The CBED disc diameter, i.e. the probe convergence, was chosen such that the discs along the direction of the smallest reciprocal lattice vector would be just touching one another.

In order to compare simulated patterns with experimental data recorded in the microscope, a series of silicon 〈111〉 pattern thickness sequences were simulated at accelerating voltages between 115 and $125 \mathrm{kV}$. The small triangle of HOLZ deficiency lines that are visible in the centers of patterns simulated at around $120 \mathrm{kV}$ are the $\{5,7, \overline{11}\}$ family. As the voltage of the simulations is increased to $122 \mathrm{kV}$, the triangle of intersection of these lines collapses to a single cross-over. Clearly, this cross-over can be used to measure the true accelerating voltage of the microscope.

Thin foils of $\langle 111\rangle$ single-crystal silicon were prepared by mechanical polishing to less than 30 $\mu \mathrm{m}$ thickness and argon-ion milling to electron transparency. The foils were loaded into a Gatan No. 673 liquid-nitrogen cold stage and then observed in the JEOL 2000FX in the North Campus Electron Microbeam Analysis Laboratory at the University of Michigan. In each experiment sample temperatures were maintained at $\sim 90 \mathrm{~K}$ in order to reduce the Debye-Waller scattering and to avoid carbon contamination of the sample. Examination of the $\langle 111\rangle$ pattern in the microscope, and adjustment of the accelerating voltage in steps as small as one tenth of a kilovolt, allowed the $\{5,7, \overline{11}\}$ family of lines to be brought 
to a cross-over. The microscope voltage at this cross-over was $121.1 \mathrm{kV}$, a deviation of less than $0.74 \%$ from the theoretical value.

Once the cross-over voltage had been reached, the accelerating voltage was held constant and a sequence of diffraction patterns were recorded at a range of thicknesses. The thickness sequence was recorded from an area of the sample that was as flat and defect-free as possible. On-line thickness measurements, performed by digitization and analysis of CBED two-beam conditions on an Apple Macintosh II computer [11], indicated that the area of the sequence varied from less than 100 to over $350 \mathrm{~nm}$.

\section{Results}

Fig. 1 shows a thickness sequence of [111] bright-field silicon CBED discs calculated at 122 $\mathrm{kV}$ in steps of $25 \mathrm{~nm}$. There are 601 pixels across the bright-field discs. As one examines each pattern of the thickness sequence one can see that most of the HOLZ lines appear to move slightly. This is most obvious at the cross-over of the $\{5,7,11\}$ family. The cross-over opens up into a triangle as the thickness increases. The movement is most clearly seen if the discs are shown as a rapid sequence of images or "movie" on the screen of a small personal computer or workstation [12]. Note that at the lower thicknesses, the width of the HOLZ lines increases, as was observed by Jones et al. [1]. The magnitude of the change in line width decreases as the thickness increases, and for silicon does not change a great deal for samples thicker than $200 \mathrm{~nm}$ [1].

Fig. 2 contains a number of experimental CBED bright-field discs from the same axis. Here too the cross-over opens into a triangle as the thickness increases. Examination of a number of other multiple HOLZ line interactions in both the experimental and theoretical patterns reveals that a number of the HOLZ lines in the patterns behave in a similar manner to the $\{5,7, \overline{11}\}$ family. These intersections are important since the changes in the dimensions of the small shapes defined by these intersections (small rhomboids, diamonds and triangles) are frequently used to determine lattice parameter and strain variations in samples in the microscope [3-6]. Clearly the movement as a function of thickness will add uncertainty to such measurements, and it is important to understand which zone axes and HOLZ lines will be thickness-sensitive in order that lattice parameter measurement may be performed under conditions where the effect is minimized. In order to explain the apparent motion of the HOLZ deficit lines, it is necessary to consider how their intensities arise.

\section{Theory}

A three-state Bloch wave model has been developed that treats the HOLZ potential as a perturbation on the basic zero-layer potential [13]. The diffracted wave amplitude for the bright-field disc at orientation $\boldsymbol{K}$ is given by:

$A_{0}(K, t)=\sum_{j} \epsilon^{j} C_{0}^{j} \exp \left(-\mathrm{i} s^{j} t / 2 k\right)$.

The $C^{j}$ and $s^{j}$ are the coefficients and transverse energies respectively of Bloch wave $j, t$ is the crystal thickness and $k$ the fast electron wavevector. For simplicity, we ignore absorption and in the absence of HOLZ diffraction the excitation amplitudes $\epsilon^{j}$ are constant and are given by $C_{0}^{j^{*}}$ To keep the model as simple as possible we assume that only two branches ( $a$ and b) are significant. In this case (1) becomes

$$
\begin{aligned}
A_{0}(K, t)= & \epsilon^{a} C_{0}^{a} \exp \left(-\mathrm{i} s^{a} t / 2 k\right) \\
& +\epsilon^{b} C_{0}^{b} \exp \left(-\mathrm{i} s^{b} t / 2 k\right) .
\end{aligned}
$$

For simple string-zone axes this two-branch approximation is often justified, particularly for orientations close to the zone axis. For example, for both Si [111] and Si [114] only branches 1 and 2 are highly excited.

In order to study the effects of HOLZ diffraction the HOLZ potential can be treated as a perturbation on the basic zero-layer potential [13]. Again for simplicity, we consider the effects of a single HOLZ state $c$, which is assumed to be a plane wave, $\exp \left[\mathrm{i}\left(\boldsymbol{K}+\boldsymbol{G}_{H}\right) \cdot \boldsymbol{R}\right]$, with transverse energy $s^{c}=\left(K+G_{H}\right)^{2} . G_{H}$ is the component of 
the HOLZ $g$-vector perpendicular top the zone axis. This three-state model is sufficient to explain the observed HOLZ line movement. As in the general HOLZ theory we analyze the intersection of the HOLZ state with one zero-layer state, which we take as state $b$. In this case $\epsilon^{a}$ is constant, $\epsilon^{b}$ and the excitation of the HOLZ state $\epsilon^{c}$ will vary with depth through the crystal, $z$. This variation is expressed as a pair of coupled differential equations [13]

$$
\begin{aligned}
& \frac{\partial \epsilon^{b}(z)}{\partial z}=\frac{\beta}{2 \mathrm{i} k} \exp [-\mathrm{i} \Phi z] \epsilon^{c}(z), \\
& \frac{\partial \epsilon^{c}(z)}{\partial z}=\frac{\beta^{*}}{2 \mathrm{i} k} \exp [\mathrm{i} \Phi z] \epsilon^{b}(z),
\end{aligned}
$$

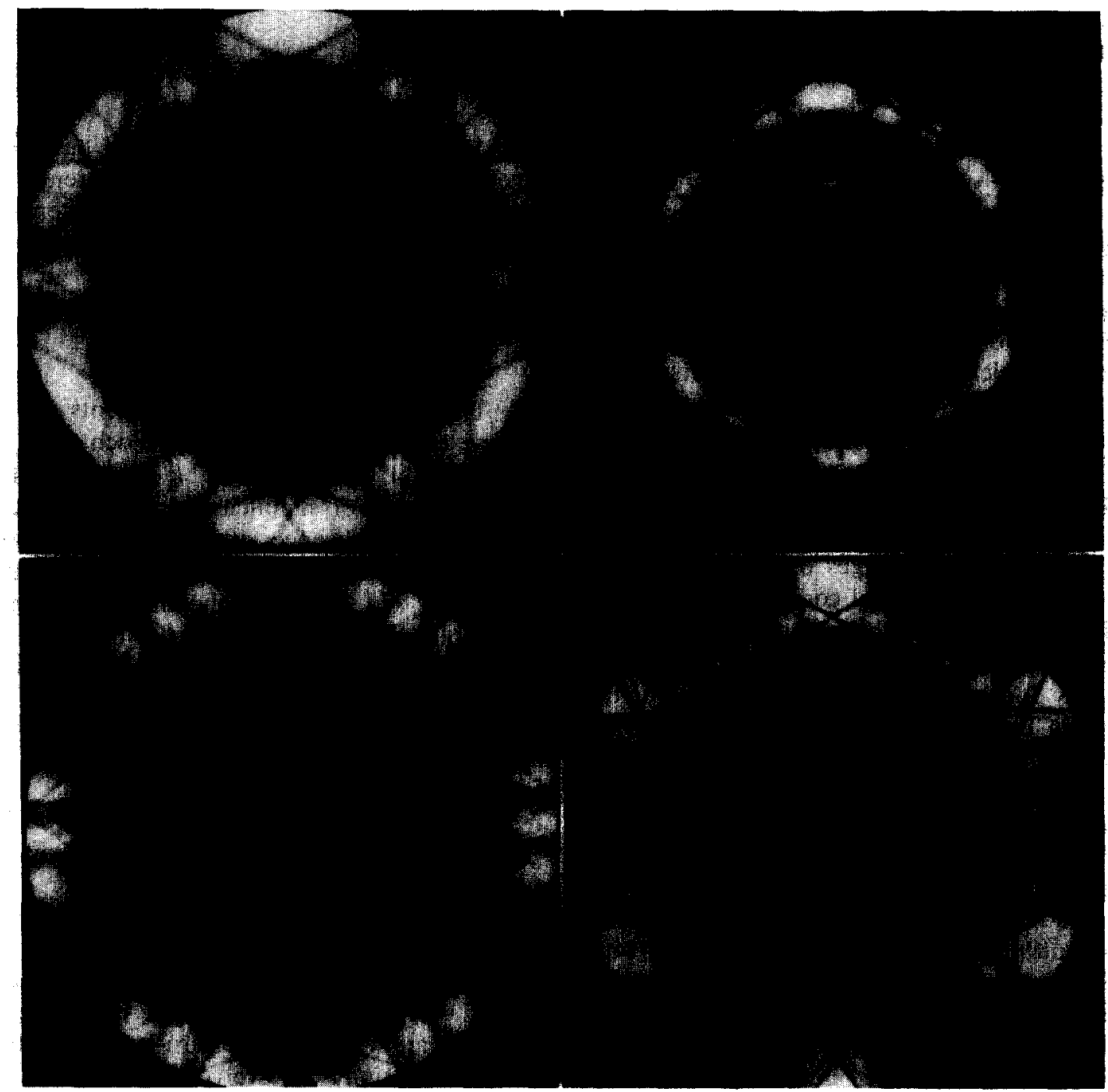

Fig. 1. Calculated $\langle 111\rangle \mathrm{Si}$ bright-field CBED discs at a series of 8 thicknesses (a-h), starting at $125 \mathrm{~nm}$ and increasing in steps of 25 to $300 \mathrm{~nm}$. There is clearly a difference in the height of the triangle of $\{5,7,11\}$ lines. Note also the changing areas of the small shapes formed by crossing HOLZ lines (an example is marked with arrows in (h)). 
where $\Phi=\left(s^{c} / 2 k-g_{z}-s^{b} / 2 k\right)$ and $\beta$ is the matrix element linking states $b$ and $c$.

These equations are solved subject to the boundary conditions that $\epsilon^{c}(0)=0$ and $\epsilon^{b}(0)=$ $C_{0}^{b^{*}}$. The solution to second order in $\beta$ (which is sufficient to analyze the form of the HOLZ line in the bright-field disc) is:

$$
\begin{aligned}
\frac{\epsilon^{b}}{C_{0}^{b^{*}}} & =1-\alpha S \\
& =1-\frac{|\beta|^{2}}{4 k^{2}} t^{2}\left[\frac{1+[\mathrm{i} \Phi t]-\exp [\mathrm{i} \Phi t]}{\Phi^{2} t^{2}}\right],
\end{aligned}
$$

where $\alpha=|\beta|^{2} t^{2} / 4 k^{2}$ and $S$ is the "shape func-

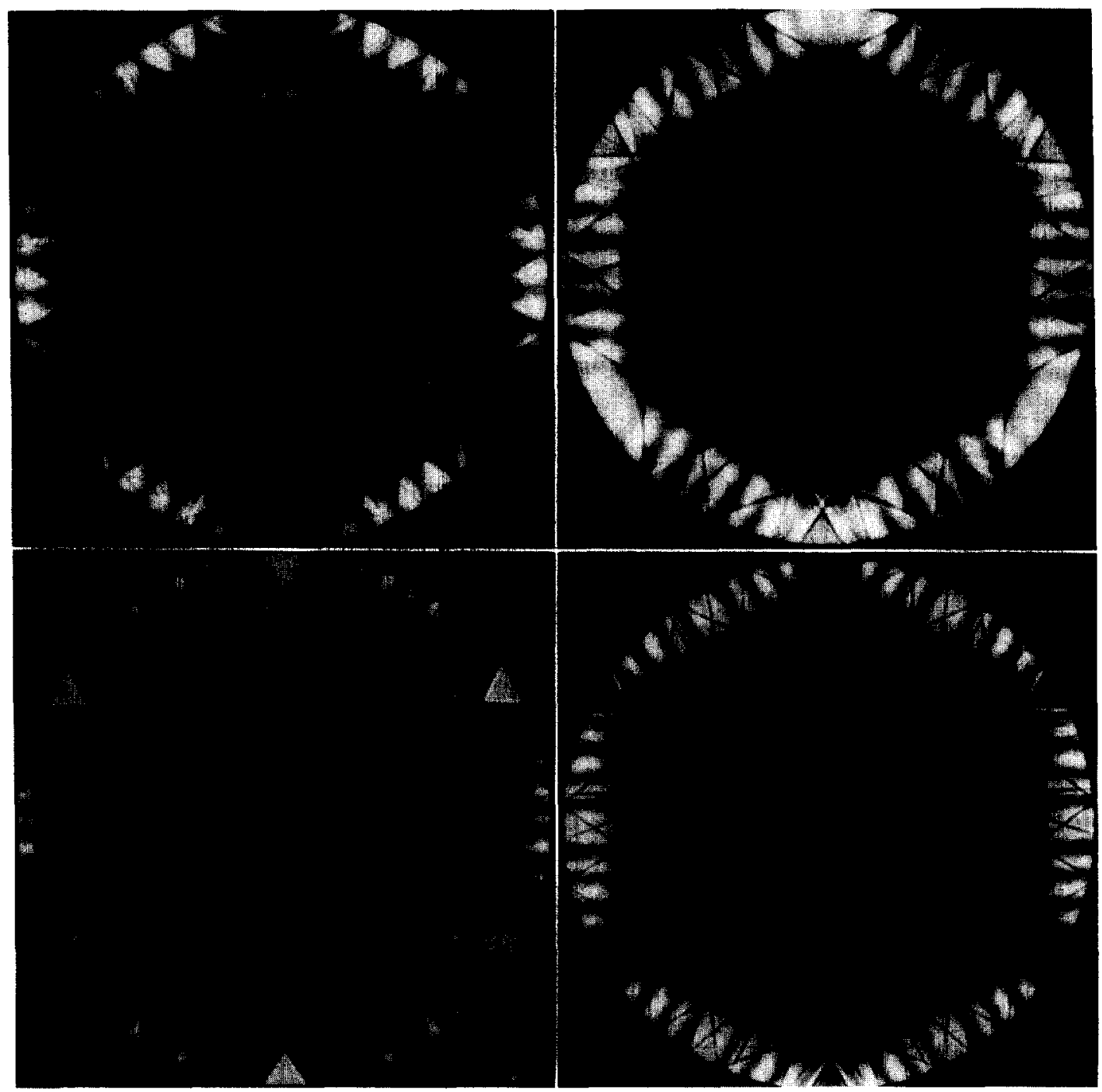

Fig. 1 (continued). 


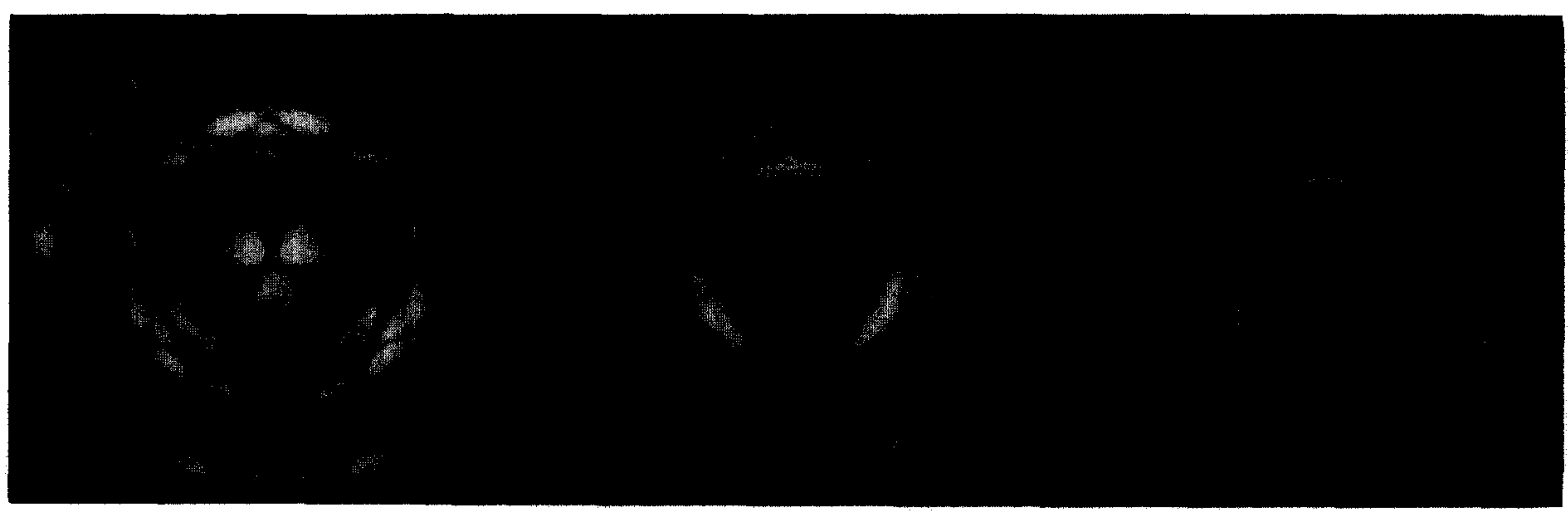

Fig. 2. Experimental $\langle 111\rangle$ Si bright-field CBED discs at three different thicknesses, the exact thicknesses are unknown; however, they are not too different from the theoretical patterns. The shift in HOLZ line positions is clearly visible at the pattern centres.

tion" associated with the HOLZ deficiency line. $\alpha$ is a measure of the basic HOLZ line strength, and for the second-order result to be valid we need $\alpha \ll 1$.

\section{Table 1}

Parameters used to calculate HOLZ deficiency line profile for Si $\langle 111\rangle$

\begin{tabular}{lc}
\hline Parameter & Value \\
\hline$R$ & 0.31 \\
$\xi$ & $262.9 \AA$ \\
$k$ & $189.3 \AA^{-1}$ \\
$G_{H}$ & $15.52 \AA^{-1}$ \\
\hline
\end{tabular}

The exact HOLZ intersection corresponds to $\Phi=0$. We consider small orientation changes $(\delta K)$ about this point such that the ZOLZ eigenvalues and eigen-vectors and the matrix element $\beta$ do not change significantly. To first order, we can then write

$$
\delta \Phi=\delta K \cdot \nabla_{K} \Phi \approx \delta K \cdot \nabla_{K} s^{c} / 2 k=\delta K G_{H} t / k
$$

where $\delta K$ is the magnitude of $\delta K$ in the direction perpendicular to the HOLZ line. The shape function $S$ is thus a function of $\left(\delta K G_{H} t / k\right)$ and so the same function can be used in all circumstances with appropriate values of $G_{H}, t$ and $k$.
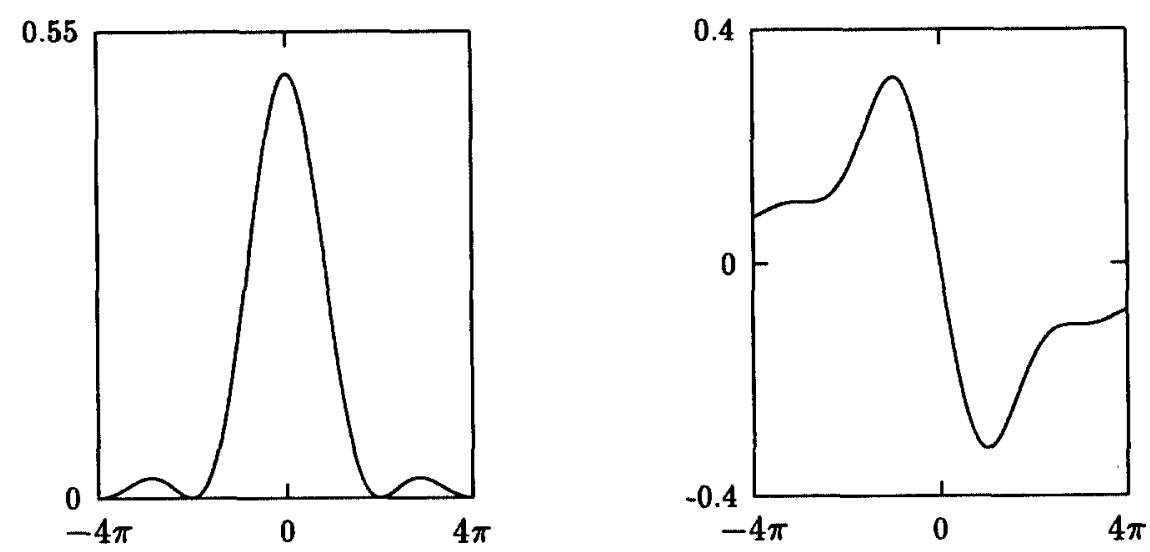

Fig. 3. (a) Real and (b) imaginary parts of the shape function $S$ in terms of the dimensionless variable $\delta \Phi=\delta K G_{H} t / k$. 
The real and imaginary parts of $S$ are plotted in fig. 3 showing that the real part is symmetric about the HOLZ line intersection while the imaginary part is anti-symmetric.

Eq. (2) now gives

$$
\begin{aligned}
A_{0}(\delta K, t)= & \left|C_{0}^{a}\right|^{2} \exp \left(-\mathrm{i} s^{a} t / 2 k\right)+\left|C_{0}^{b}\right|^{2} \\
& \times \exp \left(-\mathrm{i} s^{b} t / 2 k\right)(1-\alpha S(\delta K)),
\end{aligned}
$$

or alternatively

$$
\begin{aligned}
A_{0}(\delta K, t)= & \left|C_{0}^{a}\right|^{2} \exp \left(-\mathrm{i} s^{a} t / 2 k\right) \\
& \times\left[1+\frac{\left|C_{0}^{b}\right|^{2}}{\left|C_{0}^{a}\right|^{2}} \exp (-\mathrm{i} 2 \pi t / \xi)\right. \\
& \times(1-\alpha S(\delta K))],
\end{aligned}
$$
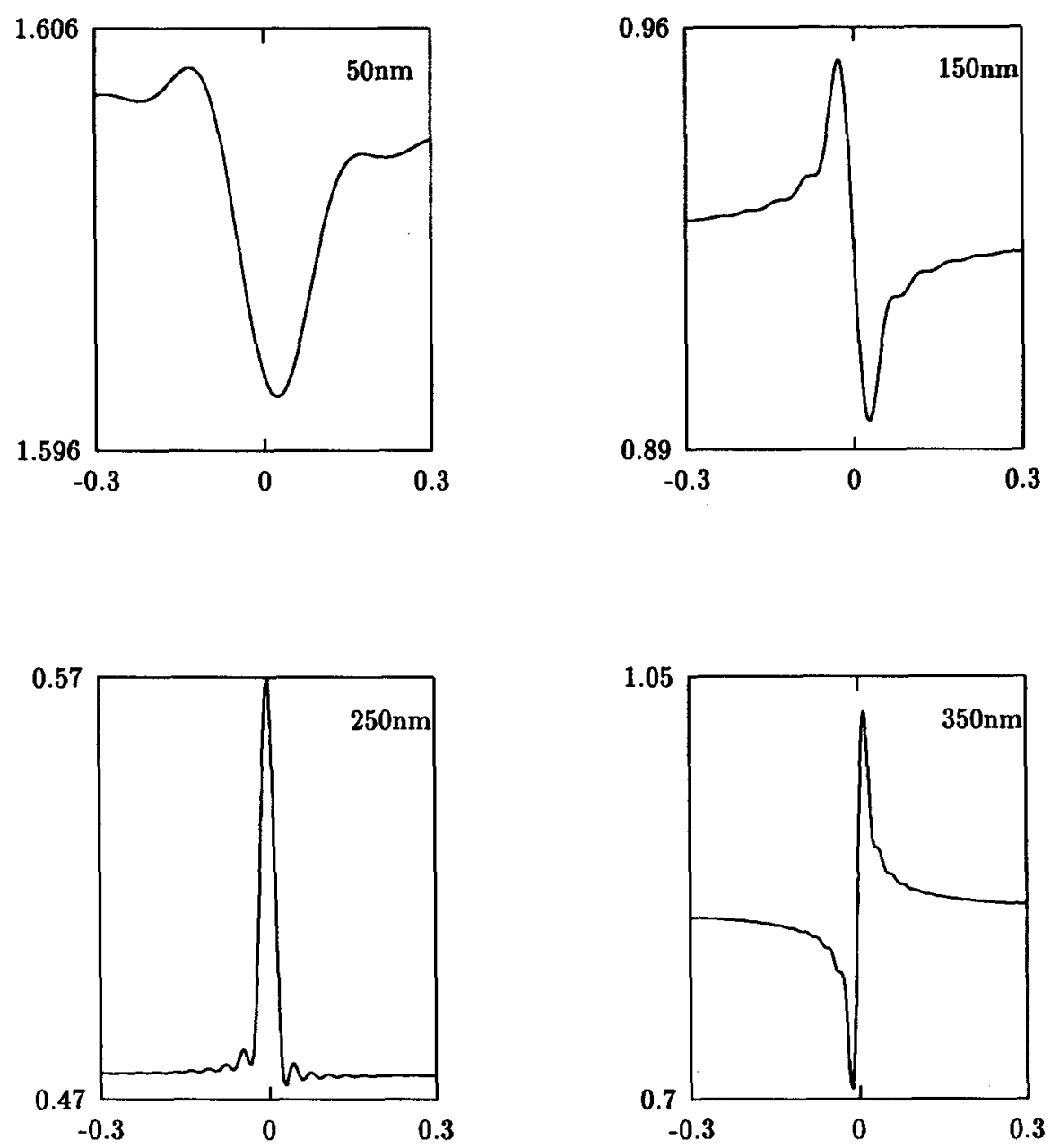

Fig. 4. Bright-field HOLZ line profiles (intensity versus $\delta K$ in $\AA^{-1}$ ) for (a) 50 , (b) 150 , (c) 250 and (d) $350 \mathrm{~nm}$. Intensity is in arbitrary units but is consistent between plots. 
where $\xi=4 \pi k /\left(s^{b}-s^{a}\right)$ is the extinction distance. The intensity is then given by

$I=\left|A_{0}\right|^{2} \propto|1+R \exp (-\mathrm{i} 2 \pi t / \xi)(1-\alpha S)|^{2}$,

where $R\left(=\left|C_{0}^{b}\right|^{2} /\left|C_{0}^{a}\right|^{2}\right)$ is the relative excitation of branches $b$ and $a$.
Expanding (8) to first order in $\alpha$ we obtain

$$
\begin{aligned}
& I \propto 1+2 R \cos (-2 \pi t / \xi)+R^{2} \\
& \quad-2 R \alpha \mathscr{R}[S \exp (-\mathrm{i} 2 \pi t / \xi)]-2 R^{2} \alpha \mathscr{R}(S),
\end{aligned}
$$

where $\mathscr{R}$ represents the real part. The first three terms in (9) provide the basic ZOLZ intensity.

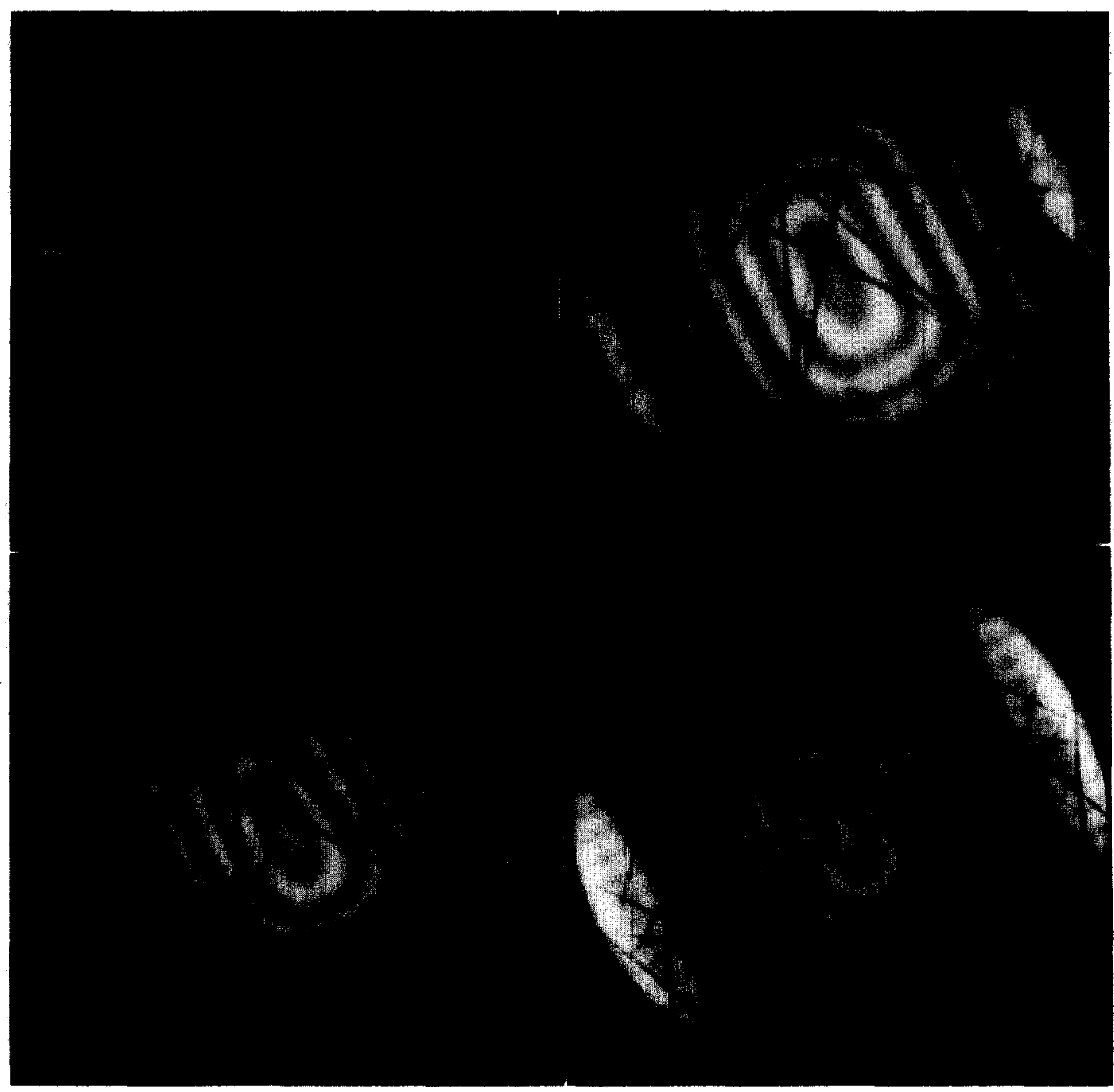

Fig. 5. Calculated (114) Si bright-field CBED discs at a series of 8 thicknesses ( $a-h$ ), starting at $125 \mathrm{~nm}$ and increasing in steps of 25 to $300 \mathrm{~nm}$. The HOLZ lines at the pattern centre are unchanged with changing thickness; however, those that interact with the zero-layer fringes do appear to shift with thickness (an example is marked with arrows in (d)). 


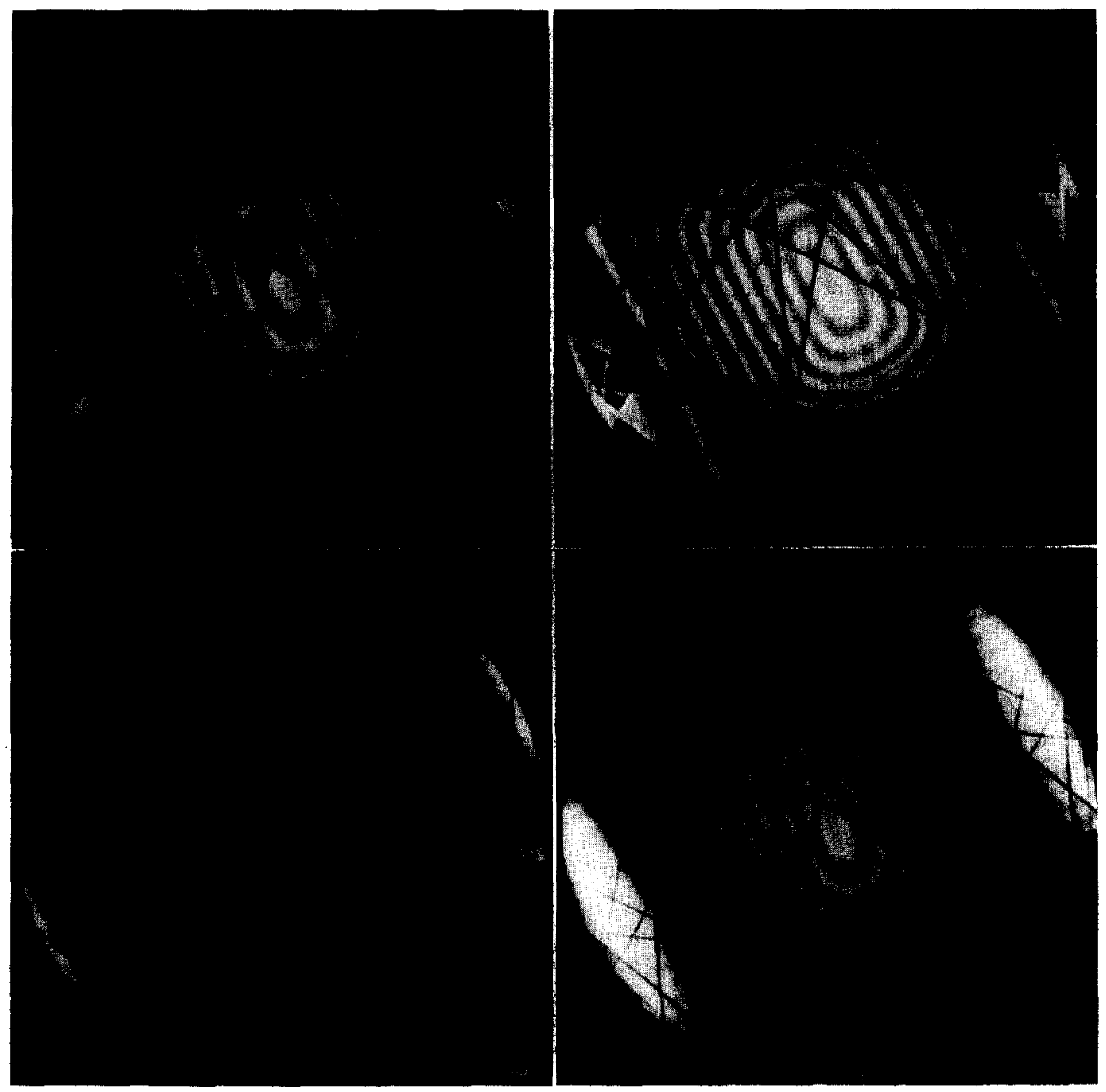

Fig. 5 (continued).

The final term (introducing only the real part of $S$ ) is an event function providing a symmetric HOLZ deficiency line, but the remaining term which describes the interference between branches $a$ and $b$ mixes in the imaginary part of the shape function. It is this term which provides the thickness dependence of the Holz line profile and, because it contains an anti-symmetric part, leads to the deficiency line appearing as a couplet of bright and dark lines. As the thickness changes the relative magnitude of the even and odd parts of $S$ vary, leading to the observed change in the HOLZ line profile and its apparent movement with thickness. It can be seen from (9) that the period of the movement will be the same as that of the underlying zero-layer contrast, namely the extinction distance for branches $a$ and $b$. From fig. 3 we can see that the maximum effect will be a movement of order two to three times the basic HOLZ line width. It is illuminating to consider two limiting cases of (9) when $R \gg 1$ and $R \ll 1$. In the former, the even $R^{2}$ term dominates lead- 
ing to a symmetric, thickness-independent HOLZ deficiency line. This corresponds to a strong perturbed branch $b$ interacting with a weak branch $a$. This, for example, would be the case for a branch 1 intersection near the zone centre of $\mathrm{Si}$ $\langle 114\rangle$ where the weak branch 2 has little effect on the branch 1 HOLZ line.

When $R \ll 1$, all the additional shape function terms become small and it becomes difficult to record any deficiency lines, e.g. branch 2 lines are not readily observed in $\mathrm{Si}\langle 114\rangle$. For the greatest thickness dependence, we need $R \approx 1$. In this case, the magnitudes of the odd and even contributions of the shape function become comparable and a coupled of highly thickness-sensitive lines will be visible. This is the effect we observe in $\mathrm{Si}\langle 111\rangle$ where branches 1 and 2 have comparable excitation.

From computed patterns of $\mathrm{Si}\langle 111\rangle$ at $122 \mathrm{kV}$ we have identified the $\{5,7,11\}$-type HOLZ lines intersecting near the axis of the bright-field disc. These HOLZ lines are intersecting with branch 2 of the zero-layer dispersion surface. A full manybeam calculation gives values for the quantities which appear in (9) (see table 1). The ratio of the two zero-layer amplitudes $R \approx 1$, which means that the interference effect will be most noticeable at this axis.

A thickness sequence of line profiles about $K=0$ is shown in fig. 4. Comparing these with the experimental results and full simulations shown earlier, a very good agreement with the theoretical model can be seen. The model clearly shows the couplet of bright and dark lines created by the interference of the two branches and also mimics the formation of the bright-only intersection at around $250 \mathrm{~nm}$.

\section{Discussion}

The apparent motion of deficit HOLZ lines in bright-field CBED discs was first reported by Rackham [14]. He noted that the crossover of the $\{\overline{55} 11\}$ family of HOLZ lines, that occurs at 103.5 $\mathrm{kV}$ at the silicon 〈111〉 zone axis, opened into small triangles as a function of thickness. Rackham attributed the motion to interaction between the zero-layer fringes and the HOLZ lines, as described by (9). In their comparisons of calculated and experimental FOLZ line profiles Jones et al. [1] also observed line shifts as a function of thickness. Microdensitometer traces, plotted as a function of thickness, across the $\{5511\}$ lines revealed that the lines moved a distance of approximately one and a half times their width. Rackham [14] deduced that if (55 11) HOLZ lines were used in lattice parameter measurements, the results would have an uncertainty of $\simeq 5 \times 10^{-4}$. This is two and a half times greater an uncertainty than is usually quoted for such measurements (typically $2 \times 10^{-4}$ ) [2]. Our analysis suggests that the apparent movement of the HOLZ lines may be as much as three times their width and therefore the uncertainty in measured lattice parameter could be as large as $1 \times 10^{-3}$.

The philosophy behind using CBED to measure lattice parameters rests on the ability to measure small changes in small volumes of material with accuracies that approach those of X-ray techniques. However, if zero-layer dynamic diffraction effects introduce large and perhaps variable uncertainties in the line positions, it is necessary to perform the measurements at zone axes that have weaker dynamic diffraction. The silicon 〈114〉 zone axis bright-field disc usually contains a zero-layer fringe pattern, although the contrast is somewhat reduced compared with the $\langle 111\rangle$ axis. Thus, for comparison, $\langle 114\rangle$ patterns were calculated at $122 \mathrm{kV}$ for a range of thicknesses. Fig. 5 is a thickness series of silicon $\langle 114\rangle$ bright-field CBED discs calculated at $122 \mathrm{kV}$, the thicknesses used for the sequence are the same as those in fig. 1. Again the effects we describe are most obvious if the thickness series is "played" as a "movie". However, careful examination reveals that the strong HOLZ lines in the centre of the 〈114〉 bright-field disc do not move appreciably with changing thickness. The centre of the pattern has a much weaker zero-layer fringe structure than either the outer edge of the disc or the $\langle 111\rangle$ bright-field disc. Examination of the lines that intersect with the stronger, well defined zero-layer fringes reveals that they oscillate in position with changing thickness in the same manner those observed at the $\langle 111\rangle$ axis. This 
thickness variation occurs towards the edge of the bright-field disc, where the excitations of branches 1 and 2 are becoming more equal. Caution must be exercised when choosing a zone axis to perform lattice parameter or strain measurements with CBED. An axis that exhibits clearly defined HOLZ lines with little or no zero-layer fringe contrast is to be preferred.

\section{Conclusions}

In summary, HOLZ deficiency lines in brightfield CBED discs of zone axes that exhibit strong zero-layer diffraction effects, e.g. strong fringe contrast, appear to move as the sample thickness is changed. The apparent movement is attributable to the interference of two strongly excited branches of the zero-layer dispersion surface with the HOLZ dispersion surface at the axis in question. The expression for the intensity of the HOLZ line contains an anti-symmetric term that is thickness dependent. The anti-symmetric term causes the HOLZ line to appear as a couplet of bright and dark lines and as the sample thickness changes the relative excitation of the bright and dark segments changes, resulting in the apparent movement of the line. As a general rule, if there is strong zero-layer fringe contrast in the bright-field disc of the zone axis pattern, then the HOLZ detail would be expected to vary in position as a function of thickness. Clearly, such axes should be avoided when trying to extract lattice constants or strain from the HOLZ line positions.

\section{References}

[1] P.M. Jones, G.M. Rackham and J.W. Steeds, Proc. Roy. Soc. (London) A 354 (1977) 197.

[2] J.W. Steeds, in: Introduction to Analytical Electron Microscopy, Eds. J.J. Hren, J.I. Goldstein and D.C. Joy (Plenum, New York, 1979) p. 387.

[3] V. Randle, I. Barker and B. Ralph, J. Electron Microsc. Tech. 13 (1989) 41.

[4] R.C. Ecob, R.A. Ricks and A.J. Porter, Scripta Met. 16 (1982) 1085.

[5] A.J. Porter, R.C. Ecob and R.A. Ricks, J. Microscopy 129 (1983) 327.

[6] J.C.H. Spence and R.W. Carpenter, in: Principles of Analytical Electron Microscopy, Eds. D.C. Joy, A.D. Romig, Jr. and J.I. Goldstein (Plenum, New York, 1986) 301.

[7] Y.P. Lin, D.M. Bird and R. Vincent, Ultramicroscopy 27 (1989) 233.

[8] J.R. Baker, PhD Thesis, University of Bristol, Bristol, UK, 1983.

[9] V.S. Sunderam, Concurrency Pract. Exp. 2(4) (1990) 315.

[10] D.M. Bird and Q.A. King, Acta Cryst. A 46 (1990) 202.

[11] J.F. Mansfield and D.C. Crawford, in: Proc. 12th Int. Congr. for Electron Microscopy, Seattle, 1990, Vol. 2, p. 504.

[12] M. Saunders, J.F. Mansfield and D.M. Bird, in: Proc. 50th Annual EMSA Meeting, 1992, to be published.

[13] D.M. Bird, J. Electron Microsc. Tech. 13 (1989) 77.

[14] G.M. Rackham, PhD Thesis, University of Bristol, Bristol, UK, 1976. 